\title{
Présentation de quelques cas de soumission chimique
}

\author{
Presentation of some cases \\ of chemical submission
}

\section{Marie-Hélène GHYSEL*, Ornella SALVADORE, Florence DESCAMPS, Salim BOUDJADA, Arnaud COUVREUR, Véronique DUPONT}

Laboratoire de Police Scientifique, 7, Boulevard Vauban - 59800 LILLE

* Auteur à qui adresser la correspondance : Marie-Hélène GHYSEL, Laboratoire de Police Scientifique, 7, Boulevard Vauban - 59800 LILLE - Tél : 33 (0)3 20128979 - Fax : 33 (0)32012 8999

(Reçu le 4 octobre 2002 ; accepté le 18 octobre 2002)

\section{$R E ́ S U M E ́$}

Pour élucider certains faits délictueux ou criminels, la justice, la police ou la gendarmerie demandent parfois des analyses toxicologiques ou des explications sur les effets de certains médicaments, dans le cadre de viol ou de vol commis au préjudice de victimes ne se souvenant plus des faits.

Nous avons été amené à traiter un certain nombre d'affaires très différentes. Celles où des médicaments neuroleptiques ont été retrouvés dans les prélèvements biologiques : bromazépam, zolpidem, et alimémazine. D'autres cas où des boissons renfermaient des médicaments : zolpidem, zopiclone et alimémazine. Des cas où de l'alcool, du cannabis et de l'ecstasy ont été retrouvés dans les prélèvements. Des explications sur les effets des médicaments nous ont été demandé lorsqu'il n'y avait pas eu de prélèvements effectués. Pour quelques affaires nous n'avons pas retrouvé de psychotropes, ou parfois de l'acide gamma hydroxy butyrique $(G H B)$ à des taux qui pouvaient être considérés comme physiologiques.

\section{MOTS-CLÉS}

Soumission chimique, analyses toxicologiques, zolpidem, zopiclone, bromazépam, alimémazine.

\section{SUMMARY}

To clarify some punishable or criminal acts, justice or police ask for toxicological analysis or explanation about effect of drugs given to victims.

Some neuroleptics have been found in biological samples: zolpidem, bromazepam, alimemazine. In other cases drugs are found in drinks: zolpidem, zopiclone, alimémazine. Some times, alcohol, cannabis or ecstasy are found. Each time we have look for gamma hydroxy butyric acid (GHB), it could be considered as physiological.

\section{KEY-WORDS}

Chemical submission, rape drugs, toxicological analysis, zolpidem, zopiclone, bromazepam, alimemazine. 


\section{Introduction}

Les analyses concernant les recherches de substances utilisées pour soumettre un individu afin de le voler ou le violer sont en augmentation.

Les affaires sont-elles plus fréquentes? ou plus souvent déclarées? Ces questions sont sans réponses, cependant les articles de presse et les émissions télévisées étant de plus en plus fréquentes sur le sujet, il est probable que l'information passe mieux auprès de tous publics et notamment des victimes mais aussi des agresseurs.

\section{Renseignements préliminaires}

Il peut arriver que nous soyons contactés par la police ou la gendarmerie dès qu'ils ont connaissance de l'affaire, avant que les prélèvements ne soient effectués. Nous insistons sur la rapidité des prélèvements et la nécessité de prélever de l'urine en plus du sang (1), voire même de la sueur s'il y a suspicion de GHB (2).

Avant de commencer les analyses un questionnaire est fourni au requérant afin d'obtenir un certain nombre de renseignements :

- Les prélèvements concernent-ils la victime ou l'inculpé?

- Date et heure des faits.

- Date et heure du prélèvement de sang et/ou d'urine.

- La victime a-t-elle déclaré avoir été endormie, ne plus se souvenir de ce qui s'est passé, avoir été sous l'effet de substances diminuant sa vigilance telles que l'alcool, des médicaments, des stupéfiants, ou un produit inconnu?

- La victime était-elle en traitement médicamenteux avant les faits, ou depuis les faits, et avec quel produit?

En fonction de la réponse à ces questions, des analyses toxicologiques ne seront effectuées que si nous sommes en mesure de trouver quelque chose avec le matériel analytique dont nous disposons.

En effet, comme rappelé par P. Kintz et col. (2), si on prend l'exemple du flunitrazépam, la présence de ses métabolites dans les urines n'est pas mise en évidence par immunoanalyse. Ces métabolites sont détectables :

- par chromatographie liquide couplée à un détecteur à barrette de diodes sur des prélèvements réalisés jusqu'à 48 heures après absorption du Rohypnol ${ }^{\circledR}$

- par chromatographie en phase gazeuse (CG-SM) par impact électronique (IE) sur des prélèvements réalisés jusqu'à 72 heures après absorption ;

- par chromatographie en phase liquide couplée à un spectromètre de masse avec ionisation chimique négative sur des prélèvements réalisés jusqu'à 96 heures après absorption ;
- par chromatographie en phase gazeuse couplée à un spectromètre de masse avec double fragmentation et ionisation chimique négative (CG-SM-SM-ICN) sur des prélèvements réalisés jusqu'à 120 heures après absorption.

Le service toxicologie du laboratoire est équipé de CGSM (IE). Donc, en fonction du laps de temps écoulé entre les faits et les prélèvements, nous pouvons être amenés à contacter le requérant afin de diriger les demandes d'analyses vers d'autres laboratoires équipés d'appareillages plus sensibles. Par ailleurs, nous conservons une partie des prélèvements pour un éventuel complément d'analyse.

\section{Protocole analytique}

Notre protocole d'analyses dans ce type d'affaires consiste généralement en une recherche générale de toxiques selon des méthodes déjà décrites (3) :

- une alcoolémie et/ou alcoolurie réalisés par CG-FID après extraction par espace de tête.

- de l'immunoanalyse pour la recherche d'opiacés, cocaïne, cannabis, amphétamine, méthamphétamine, méthadone, barbituriques, benzodiazépines, antidépresseurs tricycliques est réalisée sur les urines.

- des analyses par chromatographie en phase gazeuse couplée à la spectrométrie de masse (CG-SM) et par chromatographie liquide haute performance couplée à un détecteur à barrette de diodes (CLHP-BD). Elles sont réalisées sur les extraits préparés à partir d'urines hydrolysées par la béta glucuronidase et sur le sang après extraction à $\mathrm{pH}$ alcalin par un mélange de dichlorométhane/hexane/acétate d'éthyle. En l'absence de résultats positifs, des nouveaux essais d'extraction sont réalisés en modifiant le $\mathrm{pH}$, et/ou le solvant d'extraction et en ajoutant une dérivation préalable à la CG$S M$. Les fragments $\mathrm{m} / \mathrm{z}$ des molécules incriminées sont spécifiquement recherchés.

- des recherches d'acide gamma-hydroxybutyrique (GHB) réalisées par CG-SM, en présence d'une solution titrée de GHB-D6, utilisée comme étalon interne, après une double extraction par le chlorobutane en milieu alcalin, puis la phase aqueuse est acidifiée et extraite à pH 4,25 par l'acétate d'éthyle. La phase organique est évaporée et dérivée par du BSTFA-TMCS (Bistriméthylsilyltrifluoroacétamide $+1 \%$ de Triméthylchlorosilane).

\section{Différents types d'affaires}

De janvier 2001 à Septembre 2002, nous avons reçu une vingtaine d'affaires concernant la recherche de produits de soumission dans des inculpations pour viol, vol ou avant tentative d'homicide. Nous avions déjà présenté des cas plus anciens (4). 
La plupart des expertises ou réquisitions traitées pour ce type d'affaires nécessitait des analyses toxicologiques. Seuls, deux cas concernaient des discussions sur les effets des produits.

\section{Recherches positives}

\section{Analyses de sang et/ou d'urines}

1 - Une jeune fille se fait violer la nuit du 29 au 30 avril 2002. Elle porte plainte le 30 avril. En plus des examens et prélèvements gynécologiques, du sang et des urines sont prélevés aux fins d'analyse toxicologique. Ces prélèvements sont transmis au laboratoire le 30 avril et congelés immédiatement jusqu'à l'analyse.

La réponse au questionnaire envoyé, nous apprend que la victime a mangé des raviolis dont le goût lui a paru suspect. Elle est tombée endormie. Elle suit un traitement par Subutex ${ }^{(1)}$ et n'a pas pris de médicaments entre les faits et les prélèvements.

Effectivement, la buprénorphine et son métabolite la norbuprénorphine ont été retrouvées dans l'urine, cependant du bromazépam et du THC-COOH ont également été retrouvés dans l'urine.

Le bromazépam lui avait été donné à son insu, quant au cannabis, nous n'avons pas su si c'était également le cas, ou si elle avait omis de le déclarer.

2 - Une dame s'est fait agresser, séquestrer par des individus qui lui ont volé sa carte bleue et fait boire un liquide. Elle dit avoir vomi une partie de ce liquide sur son chemisier. Elle a fait semblant de dormir.

Des prélèvements de sang et d'urines, réalisés le lendemain des faits, ainsi que le chemisier que portait la victime au moment des faits, sont acheminés au laboratoire.

L'examen minutieux du chemisier sous des lumières de différentes longueurs d'ondes n'a pas permis de mettre en évidence de traces de vomissures. Les analyses de sang et d'urines ont mis en évidence de l'alimémazine et son métabolite dans les urines.

3 - Une jeune fille de 16 ans se retrouve dans un «état second», après avoir consommé une boisson gazeuse à base de cola.

Les urines prélevées après les faits et la boisson nous sont transmises pour analyses.

Du bromazépam, de l'ibuprofène et de la caféine sont mis en évidence dans les urines. Il n'a pas été retrouvé de produits suspects dans la boisson gazeuse.

4 - Une jeune femme est retrouvée bâillonnée devant chez elle, blessée et portant des inscriptions injurieuses au feutre sur le corps. Elle ne se souvient plus du déroulement des faits depuis qu'elle est rentrée d'une soirée au cinéma. Elle était en traitement par Lysanxia ${ }^{\circledR}$ depuis plusieurs mois.

Seul du sang a été prélevé pour analyse toxicologique.
Du nordiazépam et de l'oxazépam sont mis en évidence dans le sang. Ils peuvent provenir de la métabolisation du prazépam, principe actif du Lysanxia ${ }^{\circledR}$.

Du zolpidem a également été retrouvé dans le sang. Ce produit ne faisait pas partie du traitement de la victime et a pu provoquer l'endormissement et l'amnésie décrits.

\section{Analyses de boissons ou d'aliments}

5 - Une jeune femme a été violée par son mari et un ami de celui ci après qu'il lui ait fait absorber un liquide présent dans une fiole. La jeune femme n'a pas porté plainte dans les jours suivant les faits mais un an plus tard. Elle avait conservé le fond du contenu de la fiole qui nous a été remis pour analyse.

De l'alimémazine, de l'alimémazine sulfoxide et du zolpidem ont été retrouvés dans ce liquide. Les concentrations d'alimémazine étaient de $52,1 \mu \mathrm{g} / \mathrm{ml}$ et celle de zolpidem de $200 \mu \mathrm{g} / \mathrm{ml}$.

6 - Dans une affaire de «tentative d'empoisonnement», nous avons reçu le contenu d'une tasse à analyser. $\mathrm{Ce}$ liquide renfermait du café et de l'alimémazine et ses produits de transformation.

7 - Dans une affaire de séquestration et viol en réunion, le contenu d'une tasse a été analysé. De la zopiclone a été retrouvée dans le liquide.

8 - Une maman veut se tuer avec son enfant. Elle lui fait boire du sirop de fraise contenant un substance pour l'endormir. Du zolpidem est retrouvé dans le verre.

\section{Victimes sous influence}

Des viols ont été commis sur des personnes qui avaient leur capacité de résistance et/ou de jugement amoindries du fait de l'absorption d'alcool ou de cannabis.

Nos analyses mettent en évidence des produits mais nous ne pouvons pas savoir si les victimes ont pris les produits de leur plein gré ou sous la contrainte.

9 - Une jeune fille de 15 ans s'est fait violer en réunion, après avoir consommé du cannabis. Le requérant nous demandait de rechercher la présence de produits stupéfiants dans les urines prélevées. Du THC-COOH a été retrouvé.

10 - Une femme de 29 ans est victime d'agression sexuelle. Des prélèvements de sérum et d'urine sont réalisés aux fins de «recherche d'alcoolémie, de toxiques, et de GHB»

L'alcoolémie est de $1,35 \mathrm{~g} / \mathrm{l}$, du THC-COOH est retrouvé dans le sérum au taux de $22,7 \mathrm{ng} / \mathrm{ml}$ ( Il n'y a plus de THC). Du GHB est retrouvé dans l'urine au taux de $4 \mu \mathrm{g} / \mathrm{ml}$. Ce taux peut-être physiologique (5-7). Par ailleurs, les métabolites du propoxyphène sont retrouvés dans les urines, ainsi que de la caféine et de la cotinine.

Les recherches spécifiques de produits pouvant donner 
une soumission sont négatives.

11 - Une femme de 35 ans porte plainte pour viol. Elle suivait un traitement par Tranxène ${ }^{\circledR} 50 \mathrm{mg}$, et avait consommé 2 bières et un whisky.

Une alcoolémie de $0,81 \mathrm{~g} / \mathrm{l}$ est retrouvée 6 heures après les faits.

Il n'a pas été retrouvé de produits stupéfiants, (seule la recherche de stupéfiants semblait intéresser le requérant).

12 - Une jeune fille, victime d'un viol en réunion, porte plainte. Elle avait consommé de l'alcool et de l'ecstasy. L'alcoolémie est de 1,53 g/l, la MDMA et son métabolite la MDA sont retrouvées dans le sang aux taux respectifs de 10,9 et $2,1 \mathrm{ng} / \mathrm{ml}$

\section{Résultats négatifs}

13 - Une jeune femme de 22 ans pense avoir été violée la nuit du 31 décembre 2000 , mais ne se souvient plus de rien.

Des prélèvements sont réalisés le 2 Janvier 2001.

De la caféine et de la nicotine et leurs métabolites sont retrouvés dans les urines, ainsi que de la quinine et de la terpine, pouvant provenir de la consommation de boisson et de préparation antitussive. Il n'a pas été retrouvé de produits pouvant entraîner une soumission.

14 - Une dame d'une quarantaine d'année déclare avoir bu 2 verres de «mousseux» en début d'après-midi et de ne plus se souvenir de ce qui s'est passé. Les prélèvements de sang et d'urine sont effectués 10 heures plus tard.

L'alcoolémie sur le sang prélevé 10 heures après la consommation est encore de $0,57 \mathrm{~g} / \mathrm{l}$.

Les concentrations de GHB dans le sang et l'urine sont respectivement de 0,316 et $4,73 \mu \mathrm{g} / \mathrm{ml}$. Elles peuvent correspondre à des concentrations endogènes (5-7). Il n'a pas été retrouvé d'autres xénobiotiques que la caféine, la nicotine et la cotinine dans les prélèvements analysés.

15 - Une jeune fille a consommé des boissons alcoolisées, puis ne se souvient plus de rien.

Les prélèvements ayant été réalisés 2 jours après les faits, c'est l'urine qui est analysée. De la métoclopramide (Primpéran ${ }^{\circledR}$ ) et le métabolite du Diclofénac (Voltarène ${ }^{\circledR}$ ) ont été retrouvés. Il n'a pas été possible de savoir si ces produits avaient été pris sciemment par la victime.

\section{Demande de description des effets des produits}

16 - Une jeune fille de 17 ans est hospitalisée pour une opération chirurgicale bénigne. La veille de l'intervention, elle reçoit la visite de l'anesthésiste, il lui injecte un produit qui lui donne une sensation de brûlure dans les veines. Elle ne se souvient de rien ensuite, mais est affolée par le fait que son bas de pyjama soit retrouvé en boule au bout de son lit et suspecte avoir été violée. Aucun prélèvement biologique, ni gynécologique n'est réalisé par l'hôpital. La question était de savoir s'il existe des produits qui pouvaient donner les effets décrits.

En effet, certains produits sont décrits comme pouvant donner des douleurs plus ou moins fugace au lieu de 1 'injection, et provoquer un endormissement profond : le propofol, l'étomidate, le midazolam, le thiopental...

17 - Une jeune fille de 17 ans consomme avec 2 jeunes hommes, des bières et du cannabis. Elle aurait consommé de son plein gré, 2 joints et 6 cannettes de bière sans refuser les avances de ces messieurs. Elle se fait violer sans résistance. La question du magistrat était de savoir si les quantités consommées pouvaient influer sur son comportement.

Effectivement, l'éthanol et le cannabis modifient le comportement et les aptitudes des consommateurs. Ces effets dépendent des concentrations en principe actif, le delta 9 tétrahydrocannabinol pour le cannabis et l'éthanol pour les bières, ainsi que de la corpulence, et l'état de santé du sujet. Ils se traduisent généralement par une levée des inhibitions, de l'euphorie, une diminution des possibilités de réaction, de la griserie, de l'ébriété...

\section{Discussion et conclusions}

Les résultats de ces analyses ponctuelles, nous montrent que la nature des molécules impliquées a évolué depuis la prise de conscience des effets indésirables des benzodiazépines hypnotiques il y a une petite vingtaine d'année (8-9) et la plus grande difficulté, instaurée depuis, à se procurer du triazolam, ou du flunitrazépam.

Le zolpidem, le bromazépam et l'alimémazine sont les produits les plus souvent retrouvés dans nos affaires. Quant au GHB, il n'a pas été retrouvé de cas où il puisse être incriminé.

Les analyses réalisées dans le cadre d'affaires judiciaires ne sont qu'une partie des éléments nécessaires à la résolution d'une enquête. Nous ne connaissons que rarement les suites apportées à ces travaux.

La mise en évidence de la soumission d'un individu par un médicament donne une ampleur plus importante aux faits incriminés car il indique une préméditation, de plus elle apporte à la victime une explication au comportement qu'elle a pu avoir.

Ces quelques cas rapportés montre la variété des scénarios possibles. Les recherches négatives laissent planer un doute. Ils soulignent l'importance de la précocité et de la nature des prélèvements biologiques, ainsi que du choix des matériels analytiques utilisés. 


\section{Références}

1. Ghysel M.-H., Pépin G., Kintz P. La soumission médicamenteuse. Toxicorama, $1998 ; 10: 126-7$.

2. Kintz P., Villain M., Cirimele V., Goullé J.-P., Ludes B. Usage criminel de substances psycho-actives : le problème de la durée de détection. Acta Clinica Belgica. 2002; $57: 24-30$.

3. Ghysel M.-H., Dupont V., Descamps F., Leroux C., Salvadore $O$. Décès d'un «raveur» pouvant être imputé à un nouveau produit : la 4-méthylthioamphétamine. Ann. Toxicol. Anal. $2000 ; 11: 201-7$.

4. Ghysel M.-H., Salvadore O., Descamps F., Boudjada S., Couvreur A. La soumission chimique : de la difficulté d'appréhender l'étendue du phénomène. Présenté au $9^{\text {cme }}$ congrès de la SFTA, La Clusaz, 2001.
5. Elian A.A. Determination of endogenous gammahydroxybutyrique acid (GHB) levels in antemortem urine and blood. Forensic Sci. Int. $2002 ; 128: 120-2$.

6. Lebeau M.A., Miller M.L., Levine B. Effect of storage temperature on endogenous GHB levels in urine. Forensic Sci. Int. $2001 ; 119: 161-7$.

7. Robertson M., MacMillan B., Watson R.L., Middelberg R.A. GHB in clinical and post-mortem blood urine and serum specimens. Presented at the 29 th meeting of the Society of Forensic Toxicologists, PR 1999.

8. Poyen B., Jouglard J. Soumission médicamenteuse. Communication aux $21^{\text {èmes }}$ journées du groupement français des centres anti-poisons, Paris 1983.

9. Bismuth C., Dally S. Soumission médicamenteuse. In : Cas cliniques en toxicologie.1994. Flammarion : 82-83. 